\title{
Review on Emergence of Giant Dielectric Response in Transition Metal Doped Graphene
}

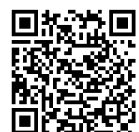

\author{
Shubhankar Mishra and Abu Jahid Akhtar* \\ Department of Physics, Diamond Harbour Women's University, Sarisha, India \\ *Corresponding author: Abu Jahid Akhtar, Department of Physics, Diamond Harbour Women's University, Sarisha, India

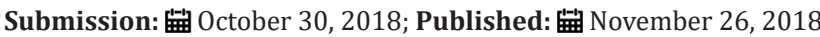

\begin{abstract}
Although, graphene has an excellent electronic and optical properties, but its dielectric property is very poor due to p-electron delocalization of graphene. From many years several strategies have been explored in order to increase its dielectric properties. In this review, we discuss the enhancement of the dielectric properties of graphene by doping transition metal in graphene. Giant value of dielectric permittivity is observed for these transition metal doped samples. We strongly believe that these materials with this extraordinary dielectric property will have enormous applications in graphene-based storage devices.
\end{abstract}

\section{Introduction}

Graphene, a two-dimensional mono layer of sp2-bonded carbon atoms, is the most promising material in the field of physics, chemistry, material science, nanoscience and nanotechnology due to its extraordinary electrical, optical, mechanical and thermal properties [1-4]. Although graphene has huge applications in electronic devices, optoelectronic devices, energy storage devices etc. [5-7] but, its dielectric properties is very poor due to p-electron delocalization of graphene. Several strategies have been taken out to improve its dielectric properties. Doping of transition metal in graphene is one of the finest method to increase the semiconducting properties of graphene as well as it has dramatic influence in dielectric properties. According to theoretical studies, the substitutional doping can modify the band structure of grapheme
[8-10]. In this review, we give a glimpse of the change in dielectric response for transition metal doped graphene and we believe that this study will be useful in fabricating graphene-based magnetodielectric devices.

\section{Structure of Transition Metal Doped Graphene} (TMG)

Graphene is an atomic-thin $2 \mathrm{D}$ carbon sheet in which the C atoms arrange in the honeycomb structure. [1,2]. The typical atomic configuration of a defect free graphene and defective graphene are shown in Figure $1 \mathrm{a} \& 1 \mathrm{~b}$, respectively. When transition metal is doped in graphene, the some of the carbon atoms in graphene is replaced by the transition metal atoms. Figure $1 \mathrm{c}$ shows the atomic configuration of transition metal doped graphene (TMG).
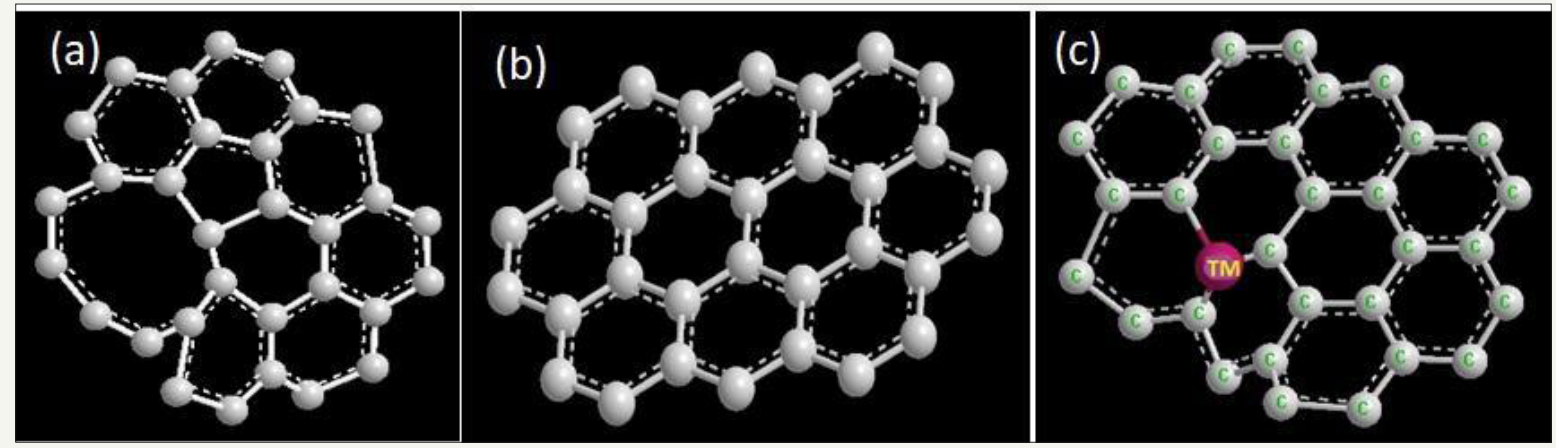

Figure 1: Typical atomic configuration of

1a: Defect free graphene,

1b: Defective graphene, and

1c: Cobalt atoms doped graphene. 


\section{Synthesis of TMG}

At first modified Hummers method [11] is used for the preparation of graphene oxide (GO). This freshly prepared of GO solution is mixed with dimethyl formamide (DMF) and sonicated for $1 \mathrm{~h}$. Aqueous solution of cobalt chloride/ nickel chloride is then added to it and the mixture is then stirred for $30 \mathrm{~min}$. At the final step, hydrazine hydrate solution is added to the mixture at $60{ }^{\circ} \mathrm{C}$ for $4 \mathrm{~h}$ under constant ultrasonic vibration and cooled to room temperature. The mixture is then filtered and washed several times with water, alcohol, and acetone to get rid of all unwanted reactants. The final product is dried in vacuum oven at $100{ }^{\circ} \mathrm{C}$ for $24 \mathrm{~h}$.

XPS study: XPS analysis have been carried out for cobalt doped graphene as shown in Figure 2 [12]. The peaks at 778.4, 793.9, and 101.4eV correspond to the B.E. of Co2p3/2, Co2p1/2, and Co 3s $1 / 2$ arising due to the presence of Co atom. All these peaks are shifed by an amount $0.4,0.6$, and $0.4 \mathrm{eV}$ respectively in respect to Co atom. These shifting indicates the doping of Co atom at vacant sites of graphene.
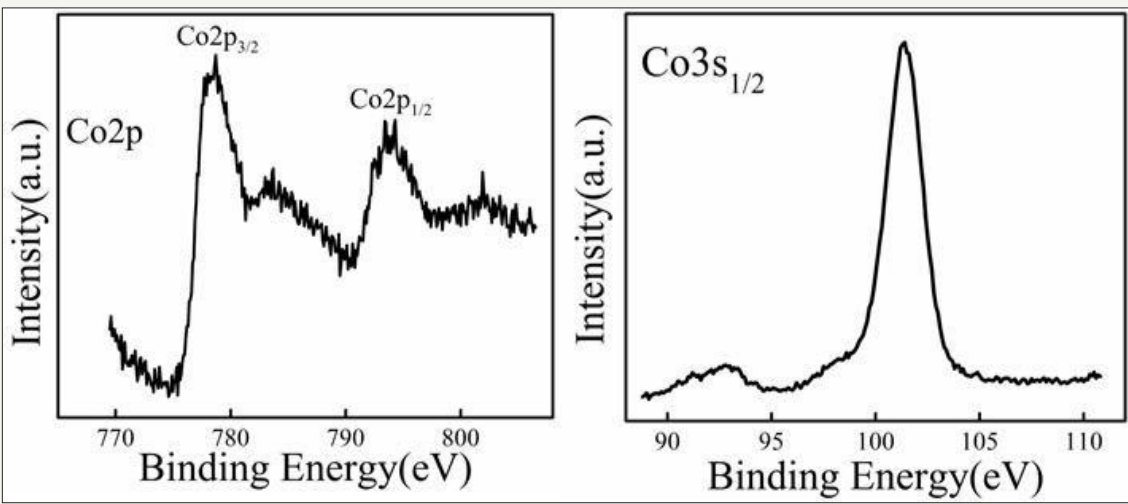

Figure 2: XPS spectra of cobalt doped graphene.

\section{Dielectric Study of TMG}

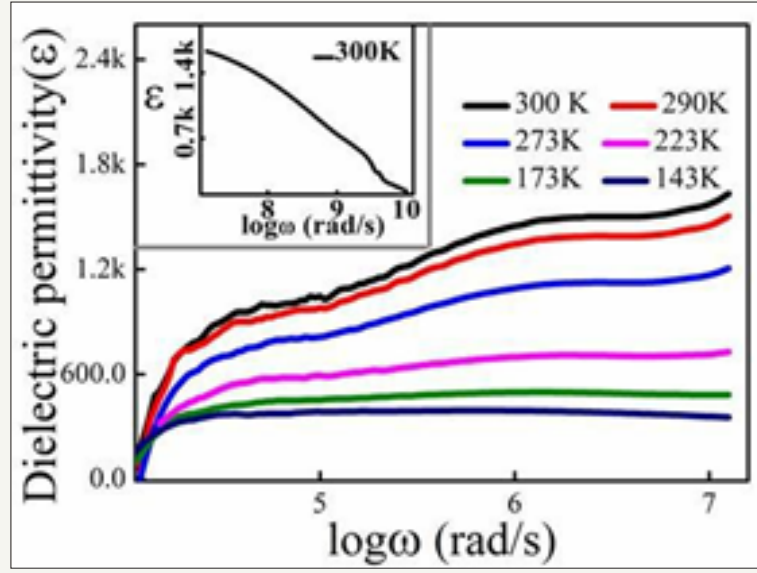

Figure 3: Variation of dielectric permittivity of Cobalt doped graphene with frequency at different temperatures.

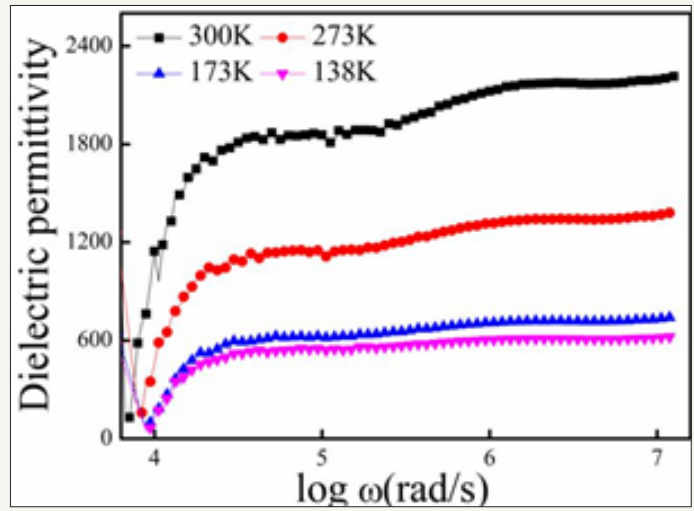

Figure 4: Variation of dielectric permittivity of nickel doped with frequency at different temperatures.
Figure 3 shows the study of dielectric permittivity as a function of frequency for the cobalt doped graphene sample at different temperatures [12]. Interesting results are observed in the Co doped sample. Firstly, very high value of dielectric permittivity $(\varepsilon \sim 2200)$ is observed for this sample at $2 \mathrm{MHz}$ frequency. Secondly, for this sample dielectric permittivity increase with increase in frequency which is quite unusual as for most of the dielectric material dielectric permittivity decrease with the increase in frequency. The same type of behavior is also observed in case of nickel doped graphene as shown in the Figure 4 [13].

This type of behaviour of dielectric permittivity is explained by the concept of trap centers. These traps are created during the doping of transition metal atoms in graphene. These trap center has a tendency to capture conduction electrons. When dc bias is applied a very few conduction electrons are only trapped in these levels while the most of trap levels remain empty. Therefore, under $\mathrm{dc}$ bias the contribution of trap capacitance is very poor. However, when ac bias is applied, the probability of electrons being captured is more due to "to and fro" motion of the electrons near the traps. With increase in frequency more numbers of electrons are captured in trap levels and permittivity increases with frequency as shown in Figure 3 . When all the traps are filled by the conduction electrons the capacitance becomes saturated and finally permittivity value starts to decrease with frequency as in case of usual dielectric material.

Another important observation in the dielectric study for both the samples Figure $3 \& 4$ is that the decrease of permittivity value with decreases in temperature. It is well known that the mobility of electron is inversely proportional to temperature so at low temperature the mobility of electrons is high enough. As a result, at low temperature the probability of the electrons being trapped 
are less and hence the permittivity value decreases with decrease in temperature.

\section{Conclusion}

In summary, transition metal doped graphene emerges as a new class of materials which show giant dielectric permittivity as well as unsual dielectric behaviour where permittivity value increases with frequency. The high value of dielectric permittivity and the unusual behaviour are explained on the concept of trap states. We strongly believe that these materials with this extraordinary dielectric property will have enormous applications in graphenebased storage devices.

\section{References}

1. Chen JH, Li L, Cullen WG, Williams ED, Fuhrer MS (2011) Tunable Kondo effect in graphene with defects. Nat Phys 7: 535-538.

2. Berger C, Song Z, Li X, Wu X, Brown N, et al. (2006) Electronic confinement and coherence in patterned epitaxial graphene. Science 312:1191-1196

3. Lee C, Wei X, Kysar JW, Hone J (2008) Measurement of the elastic properties and intrinsic strength of monolayer graphene. Science 321(5887): 385-388

4. Geim AK, Novoselov KS (2007) The rise of graphene. Nat Mater 6: 183191.
5. Zhang B, Cui T (2011) An ultrasensitive and low-cost graphene sensor based on layer-by-layer nano self-assembly. Appl Phys Lett 98(7): 073116

6. Cheng Z, Li Q Li Z, Zhou Q Fang Q (2010) Suspended graphene sensors with improved signal and reduced noise. Nano Lett 10(5): 1864-1868.

7. Yoo E, Kim J, Hosono E, Zhou HS, Kudo T, et al. (2008) Large reversible Li storage of graphene nanosheet families for use in rechargeable lithium ion batteries. Nano Lett 8(8): 2277-2282.

8. Wang X, Li X, Zhang L, Yoon Y, Weber PK, et al. (2009) N-doping of graphene through electrothermal reactions with ammonia. Science 324(5928): 768-771.

9. Zhang C, Fu L, Liu N, Liu M, Wang Y, et al. (2011) Synthesis of nitrogendoped graphene using embedded carbon and nitrogen sources. Adv Mater 23(8): 1020-1024

10. Lin YC, Lin CY, Chiu PW (2010) Controllable graphene N-doping with ammonia plasma. Appl Phys Lett 96: 133110.

11. Hummers WS, Offeman RE (1958) Preparation of graphitic oxide. J Am Chem Soc 80(6): 1339.

12. Akhtar AJ, Gupta A, Shaw BK, Saha SK (2013) Unusual dielectric response in cobalt doped reduced graphene oxide. Appl Phys Lett 103: 242902

13. Akhtar AJ, Gupta A, Saha SK (2015) Trap induced tunable unusual dielectric properties in transition metal doped reduced graphene oxide. RSC Advances 5: 9594.
Creative Commons Attribution 4.0

International License

For possible submissions Click Here

\section{Submit Article}

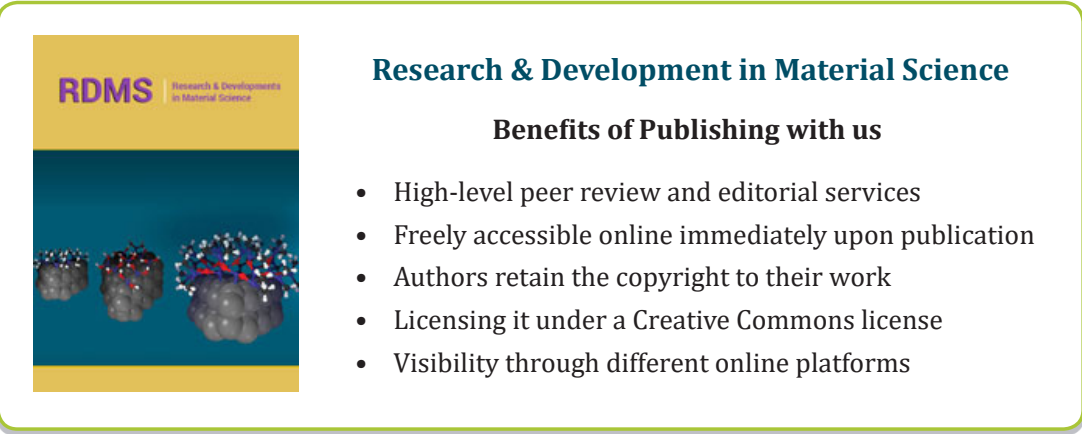

